\title{
High expression of myocyte enhancer factor 2C (MEF2C) is associated with adverse-risk features and poor outcome in pediatric acute myeloid leukemia: a report from the Children's Oncology Group
}

George S. Laszlo', Todd A. Alonzo 2,3, Chelsea J. Gudgeon', Kimberly H. Harrington', Alex Kentsis 4,5,6, Robert B. Gerbing ${ }^{3}$, Yi-Cheng Wang ${ }^{3}$, Rhonda E. Ries ${ }^{1}$, Susana C. Raimondi ${ }^{3,7}$, Betsy A. Hirsch ${ }^{3,8}$, Alan S. Gamis ${ }^{3,9}$, Soheil Meshinchi $i^{1,3,10}$ and Roland B. Walter ${ }^{1,11,12^{*}}$

\begin{abstract}
Background: Recent studies have identified myocyte enhancer factor 2C (MEF2C) as cooperating oncogene in acute myeloid leukemia (AML) and suggested a contribution to the aggressive nature of at least some subtypes of AML, raising the possibility that MEF2C could serve as marker of poor-risk AML and, therefore, have prognostic significance.

Methods: To test this hypothesis, we retrospectively quantified MEF2C expression in pretreatment bone marrow specimens in participants of the AAML0531 trial by reverse-transcriptase polymerase chain reaction and correlated expression levels with disease characteristics and clinical outcome.

Results: In all 751 available patient specimens, MEF2C messenger RNA (mRNA) was detectable and varied >3000-fold relative to $\beta$-glucuronidase. Patients with the highest relative MEF2C expression (4th quartile) less likely achieved a complete remission after one course of chemotherapy than the other patients ( $67 \mathrm{vs.} 78 \%, P=0.005$ ). They also had an inferior overall survival ( $P=0.014$; at 5 years $55 \pm 8$ vs. $67 \pm 4 \%)$, inferior event-free survival $(P<0.001$; at 5 years $38 \pm 7$ vs. $54 \pm 4 \%)$, and higher relapse risk than patients within the lower 3 quartiles of MEF2C expression $(P<0.001$; at 5 years $53 \pm 9$ vs. $35 \pm 5 \%$ ). These differences were accounted for by lower prevalence of cytogenetically/molecularly defined low-risk disease (16 vs. $46 \%, P<0.001)$ and higher prevalence of standard-risk disease (68 vs. $42 \%, P<0.001)$ in patients with high MEF2C expression, suggesting that MEF2C cooperates with additional pathogenic abnormalities.

Conclusions: High MEF2C expression identifies a subset of AML patients with adverse-risk disease features and poor outcome. With confirmation that high MEF2C mRNA expression leads to overexpression of MEF2C protein, these findings provide the rationale for therapeutic targeting of MEF2C transcriptional activation in AML.
\end{abstract}

Keywords: AAML0531, Acute myeloid leukemia (AML), Adverse risk, Biomarker, Children's Oncology Group (COG), Myocyte enhancer factor 2C (MEF2C), Pediatric, Transcription factor

\footnotetext{
*Correspondence: rwalter@fredhutch.org

${ }^{1}$ Clinical Research Division, Fred Hutchinson Cancer Research Center, 1100

Fairview Ave N, D2-190, Seattle, WA 98109-1024, USA

${ }^{11}$ Department of Medicine, Division of Hematology, University of

Washington, Seattle, WA, USA

Full list of author information is available at the end of the article
}

C Biomed Central

(c) 2015 Laszlo et al. Open Access This article is distributed under the terms of the Creative Commons Attribution 4.0 International License (http://creativecommons.org/licenses/by/4.0/), which permits unrestricted use, distribution, and reproduction in any medium, provided you give appropriate credit to the original author(s) and the source, provide a link to the Creative Commons license, and indicate if changes were made. The Creative Commons Public Domain Dedication waiver (http://creativecommons.org/publicdomain/zero/1.0/) applies to the data made available in this article, unless otherwise stated. 


\section{Background}

Myocyte enhancer factor 2 (MEF2) proteins, composed of four family members in vertebrates, are transcription factors that were initially studied in the control of muscle development [1]. In particular, gene deletion studies in mice identified essential functions of $M E F 2 C$ in cardiac myogenesis and right ventricular development [2]. However, subsequent studies have indicated that $M E F 2 C$ plays a much broader biological role and is involved in the function and generation of tissues other than cardiac and skeletal muscle, including bone development and osteoclast-mediated bone resorption, neuronal development, and craniofacial and melanocyte development [3].

Increasing evidence also suggests an important role of $M E F 2 C$ in the normal hematopoietic system, particularly for the production of immature and mature lymphoid cells and as a modulator of the cell fate decision between monocyte and granulocyte differentiation [3-6]. This is indicated by genetic studies in mice showing that Mef2c deficiency is associated with reduced levels of monocytes in response to cytokines [4] as well as profound defects in the production of B cells, T cells, natural killer cells, and common lymphoid progenitor cells, as well as enhanced myeloid output [5]. In turn, constitutive expression of $M e f 2 c$ in the bone marrow results in increased monopoiesis at the expense of granulopoiesis [4]. In human acute myeloid leukemia (AML) cell line models, 1,25-dihydroxyvitamin D3 induces monocytic differentiation and CD14 expression, an effect that is mediated through activation of $M E F 2 C$ signaling via regulation of CCAAT-/enhancer-binding protein alpha (CEBPA) [6]. Consistent with these central functions, $M E F 2 C$ has been found to be aberrantly expressed in subsets of $\mathrm{T}$ cell acute lymphoblastic leukemia (T-ALL) and in early thymocyte precursor (ETP) T-ALL in particular, an aggressive leukemia that tends to be refractory to chemotherapy and shares genetic features with AML [7-10]. In $\mathrm{AML}, M E F 2 C$ has been found to be overexpressed in distinct molecular subsets of adult onset AML, including mixed lineage leukemia $(M L L)$ gene-rearranged and ectropic virus integration site 1 (EVI1)-overexpressing leukemias [11]. Models of both MLL and EVI1 leukemias have been, and continue to be, instrumental in our understanding of fundamental principles of leukemogenesis and the identification of pathways that confer tumor aggressiveness and resistance to chemotherapy [12-21]. Functional studies using mouse leukemia models demonstrate that $M e f 2 c$ is a potent oncogene, causing fully penetrant AML in cooperation with SOX4 [11, 22, 23]. In addition, $M e f 2 c$ is required for the growth of mouse leukemias induced by $M L L-A F 9$ [11].

Together, these studies suggest that $M E F 2 C$ participates in key molecular mechanisms of AML pathogenesis and could serve as a marker of poor-risk AML and, therefore, have prognostic significance. Here, we tested this hypothesis by retrospectively quantifying $M E F 2 C$ expression in pretreatment bone marrow specimens and by associating $M E F 2 C$ expression level with disease characteristics and outcome in participants of the Children's Oncology Group (COG) AML protocol, AAML0531 (NCT00372593). AAML0531 was a multicenter, randomized phase 3 study, which found that the addition of gemtuzumab ozogamicin to intensive chemotherapy improved the event-free survival (EFS) through reduction of the relapse risk (RR) relative to intensive chemotherapy alone in patients aged $<30$ years with newly diagnosed de novo non-APL AML, excluding those with bone marrow failure syndromes, juvenile myelomonocytic leukemia, or Down syndrome (if $\leq 3$ years of age) between 2006 and 2010 [24].

\section{Results}

Identification of MEF2C expression as predictive biomarker in participants of AAML0531

Among the 1022 eligible patients enrolled in AAML0531, 980 (96 \%) consented to have diagnostic bone marrow specimens banked for future cancer research. At the time this research was conducted, RNA was available from 765 patients. Fourteen samples were excluded because of inadequate RNA as determined by low $\beta$-glucuronidase (GUSB) expression $(\mathrm{Ct}>33.09)$. The remaining 751 patients $(77 \%)$ were used for quantitation of $M E F 2 C$ expression levels. In all of these specimens, $M E F 2 C$ mRNA was detectable and varied $>3000$-fold relative to GUSB mRNA (0.0091-29.1272 [median 0.7978]; Fig. 1).

\section{Association between MEF2C expression and clinical outcome}

Studying the relationship between $M E F 2 C$ expression and clinical outcome, we initially analyzed patient outcomes per quartile of $M E F 2 C$ expression and noticed that the 188 patients with the highest relative $M E F 2 C$ expression (4th quartile, corresponding to an expression of $\geq 1.66$ relative to GUSB) fared worse than the 563 patients in the 1 st, 2nd, or 3rd quartiles of $M E F 2 C$ expression, respectively, with little difference between the first 3 quartiles. We therefore subsequently compared patients with the highest relative $M E F 2 C$ expression (4th quartile) to patients with lower expression (1st to 3rd quartile).

Analyzing responses to initial chemotherapy, we found that patients with high relative $M E F 2 C$ expression were statistically significantly less likely to have achieved a complete remission (CR) after one course of chemotherapy than patients with lower $M E F 2 C$ expression (67 vs. $78 \%, P=0.005$ ) and tended to be more likely to have flow cytometrically detectable minimal residual disease (MRD) at the end of the first induction course (33 vs. $27 \%$, $P=0.132$ ). Some patients with high $M E F 2 C$ expression 

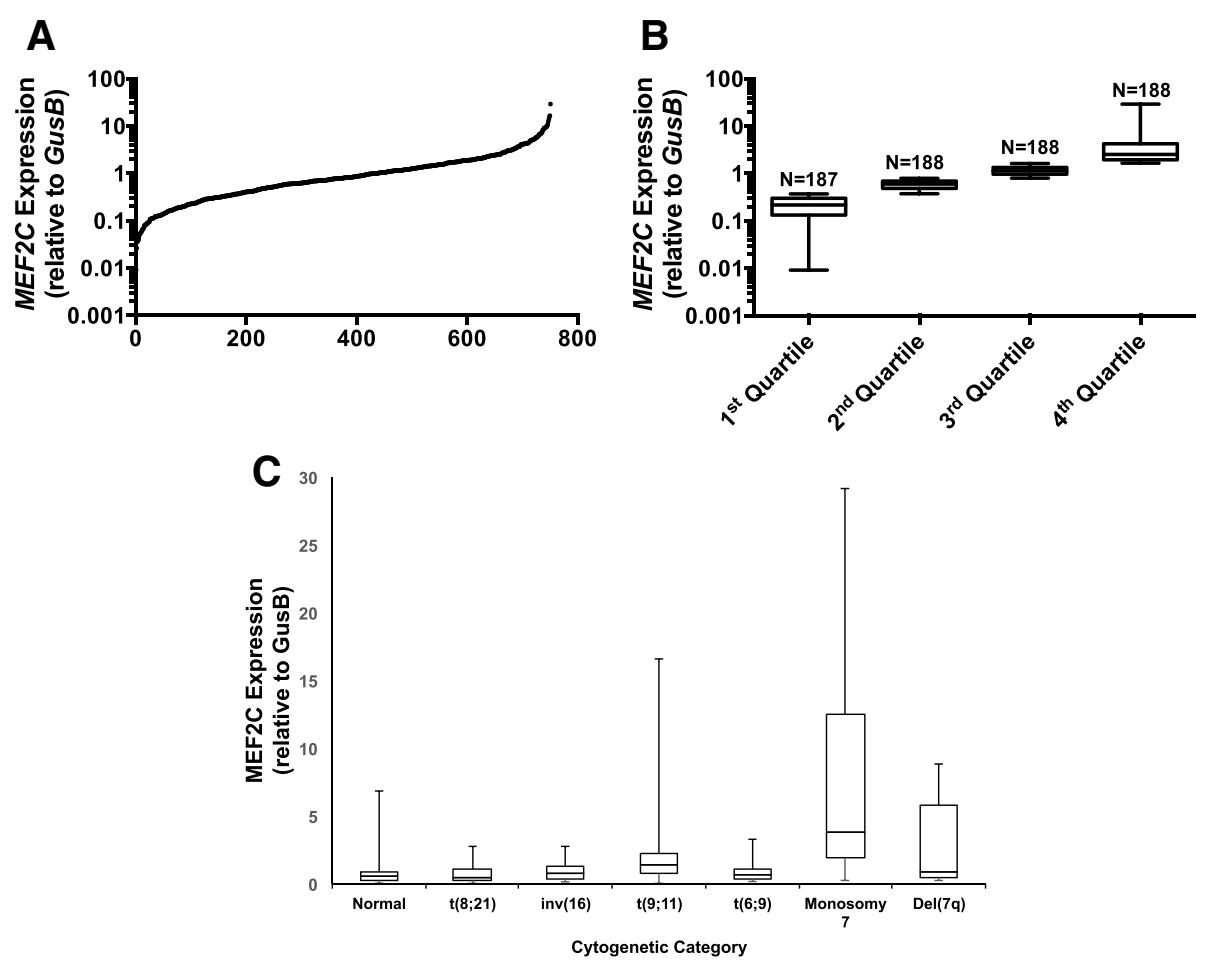

Fig. 1 MEF2C expression in AAML0531. Quantitative expression of MEF2C relative to beta glucuronidase (GUSB) in diagnostic bone marrow specimens from the 751 patients who were included in this study. a Relative MEF2C expression across the entire study cohort. b Distribution of relative MEF2C expression across quartiles of MEF2C expression. c Distribution of relative MEF2C expression across individual cytogenetic categories

were able to achieve remission with re-induction therapy, and the proportion of patients with high $M E F 2 C$ expression in CR after two courses of induction chemotherapy approached that of patients with low $M E F 2 C$ expression (86 vs. $90 \%, P=0.102$ ). We subsequently evaluated how $M E F 2 C$ expression related to parameters of long-term outcome and found that patients with the highest $M E F 2 C$ expression had an inferior overall survival (OS; $P=0.014$; at 5 years $55 \pm 8$ vs. $67 \pm 4 \%)$, inferior EFS $(P<0.001$; at 5 years $38 \pm 7$ vs. $54 \pm 4 \%$ ), and higher RR than the patients within the lower 3 quartiles of $M E F 2 C$ expression $(P<0.001$; at 5 years $53 \pm 9$ vs. $35 \pm 5 \%$; Table 1 and Fig. 2a-c). Of note, exploratory multiple cutpoint analyses for OS and EFS indicated that the most statistically significant results were centered around the Q4 cutpoint region, supporting our approach of comparing patients with the highest quartile of relative $M E F 2 C$ expression with those having lower relative $M E F 2 C$ expression (data not shown).

We next performed subgroup analyses to investigate the association between $M E F 2 C$ expression and outcome in specific risk groups; these studies were of exploratory nature since our ability to perform these analyses was relatively limited given the sample size of the individual risk groups. As summarized in Table 1, the association between high $M E F 2 C$ expression and increased risk of relapse and, consequently, lower EFS was particularly apparent in the subset of cytogenetically/molecularly defined low-risk patients, whereas no strong trend was seen in patients with standard-risk or high-risk patients.

\section{Association between MEF2C expression and characteristics of study population}

To investigate associations between relative $M E F 2 C$ expression and demographics, baseline laboratory findings, and pretreatment characteristics of the study cohort, we compared patients with high $M E F 2 C$ expression (4th quartile) with those having low $M E F 2 C$ expression (1st to 3rd quartile). As summarized in Table 2, patients with high $M E F 2 C$ expression were younger $(P<0.001)$ and more likely presented with hepatomegaly $(P=0.006)$ or splenomegaly $(P<0.001)$. They also had a slightly but statistically significantly higher percentage of bone marrow blast at diagnosis. In contrast, there was no statistically significant difference in gender distribution, white blood cell (WBC) count, or hemoglobin between patients with high and low $M E F 2 C$ expression. Importantly, however, $M E F 2 C$ expression was strongly associated with cytogenetic and molecular abnormalities. Specifically, patients with high $M E F 2 C$ expression less likely had CBF translocations (inv(16): $P=0.007$ and $\mathrm{t}(8 ; 21): P<0.001$ ) or normal karyotype AML $(P<0.001)$; conversely, they were more likely to have leukemias with monosomy 7 
Table 1 Comparison of treatment responses of patients with low (Q1-3) vs. high (Q4) MEF2C expression

\begin{tabular}{|c|c|c|c|c|c|}
\hline \multirow[t]{2}{*}{ Outcome at 5 years } & \multicolumn{4}{|c|}{ Relative MEF2C expression } & \multirow[t]{2}{*}{$P$ value* } \\
\hline & Low (Q1-3) & High (Q4) & Hazard ratio $^{a}$ & $95 \%$ confidence interval & \\
\hline All patients & $n=563$ & $n=188$ & & & \\
\hline OS & $67 \pm 4 \%$ & $55 \pm 8 \%$ & 1.385 & $1.07-1.80$ & 0.014 \\
\hline EFS & $54 \pm 4 \%$ & $38 \pm 7 \%$ & 1.510 & $1.21-1.88$ & $<0.001$ \\
\hline RR & $35 \pm 5 \%$ & $53 \pm 9 \%$ & 1.813 & $1.36-2.42$ & $<0.001$ \\
\hline Low-risk patients & $n=255$ & $n=29$ & & & \\
\hline OS & $81 \pm 5 \%$ & $76 \pm 20 \%$ & 1.433 & $0.43-4.82$ & 0.561 \\
\hline EFS & $69 \pm 6 \%$ & $51 \pm 20 \%$ & 1.597 & $0.90-2.82$ & 0.104 \\
\hline RR & $23 \pm 6 \%$ & $49 \pm 20 \%$ & 2.290 & $1.26-4.17$ & 0.011 \\
\hline Standard-risk patients & $n=230$ & $n=123$ & & & \\
\hline OS & $55 \pm 7 \%$ & $55 \pm 9 \%$ & 0.992 & $0.71-1.38$ & 0.961 \\
\hline EFS & $42 \pm 7 \%$ & $38 \pm 9 \%$ & 1.143 & $0.86-1.52$ & 0.356 \\
\hline RR & $49 \pm 8 \%$ & $55 \pm 11 \%$ & 1.270 & $0.88-1.83$ & 0.201 \\
\hline High-risk patients & $n=69$ & $n=30$ & & & \\
\hline OS & $52 \pm 12 \%$ & $37 \pm 10 \%$ & 1.452 & $0.81-2.59$ & 0.204 \\
\hline EFS & $31 \pm 11 \%$ & $29 \pm 17 \%$ & 1.155 & $0.70-1.92$ & 0.576 \\
\hline RR & $46 \pm 16 \%$ & $47 \pm 27 \%$ & 1.381 & $0.59-3.22$ & 0.446 \\
\hline
\end{tabular}

*Log-rank $P$ value

${ }^{a}$ Estimates from Weibull parametric models

$(P<0.001)$ and abnormalities involving 11q23 $(P<0.001)$. Furthermore, patients with high $M E F 2 C$ less likely had a FLT3/ITD $(P=0.018)$ or a mutation in either NPM1 $(P=$ $0.010)$ or $C E B P A(P=0.002)$. Consistently, patients with high $M E F 2 C$ expression less likely had low-risk disease (16 vs. $46 \%, P<0.001)$ and more likely had standard-risk disease ( 68 vs. $42 \%, P<0.001$ ) than those with lower $M E F 2 C$ expression (Table 2).

\section{MEF2C expression as an independent predictive factor}

Finally, we evaluated the potential role of $M E F 2 C$ expression as an independent predictor of OS, EFS, and $\mathrm{RR}$ in regression models (Table 3). Given the strong association between disease risk and $M E F 2 C$ expression, one might attribute the worse outcome for patients with high $M E F 2 C$ expression to the lower prevalence of leukemias with more favorable prognoses in this subgroup. Indeed, after adjustment for disease risk, age, FAB category, and treatment arm, high $M E F 2 C$ expression was no longer statistically significantly associated with inferior OS (HR $=0.99$ [0.72-1.36], $P=0.929)$, inferior EFS $(\mathrm{HR}=$ $1.14[0.86-1.49], P=0.365)$, or higher $R R(H R=1.32$ [0.91-1.92], $P=0.137$; Table 3).

\section{Discussion}

Recent studies have highlighted a possible role of $M E F 2 C$ in the molecular pathogenesis and therapy response of AML [3]. Using over 750 pretreatment bone marrow specimens from pediatric patients enrolled in a recent cooperative group phase 3 trial, ours is the first study to quantify $M E F 2 C$ mRNA abundance by RT-PCR and comprehensively examine the relationship between $M E F 2 C$ expression and disease characteristics as well as treatment outcome in pediatric AML. The findings from these investigations support three main conclusions. First, $M E F 2 C$ is widely expressed in pediatric AML, with relative levels that vary considerably ( $>3000$-fold) across bone marrows of patients with active disease. Second, high $M E F 2 C$ expression is associated with adverse treatment outcome in pediatric AML. Specifically, in our cohort, patients with the highest relative $M E F 2 C$ expression (4th quartile) less likely achieved a CR after one course of chemotherapy than the other patients; they also had an inferior OS and EFS and higher RR than patients within the lower 3 quartiles of $M E F 2 C$ expression. And third, high $M E F 2 C$ expression is associated with several adverserisk features. Specifically, in participants of AAML0531, high relative expression of $M E F 2 C$ was associated with a lower prevalence of cytogenetically/molecularly defined low-risk disease and higher prevalence of standard-risk disease, largely because of a lower prevalence of CBF leukemias or mutations in NPM1 or CEBPA and a higher prevalence of leukemias with monosomy 7 or abnormalities involving 11q23. Conversely, high relative expression of $M E F 2 C$ was associated with some better risk features, particularly a lower prevalence of FLT3/ITD (10 vs. $18 \%$; Table 2). Still, the associations between adverse cytogenetic or molecular disease risk features with high $M E F 2 C$ 


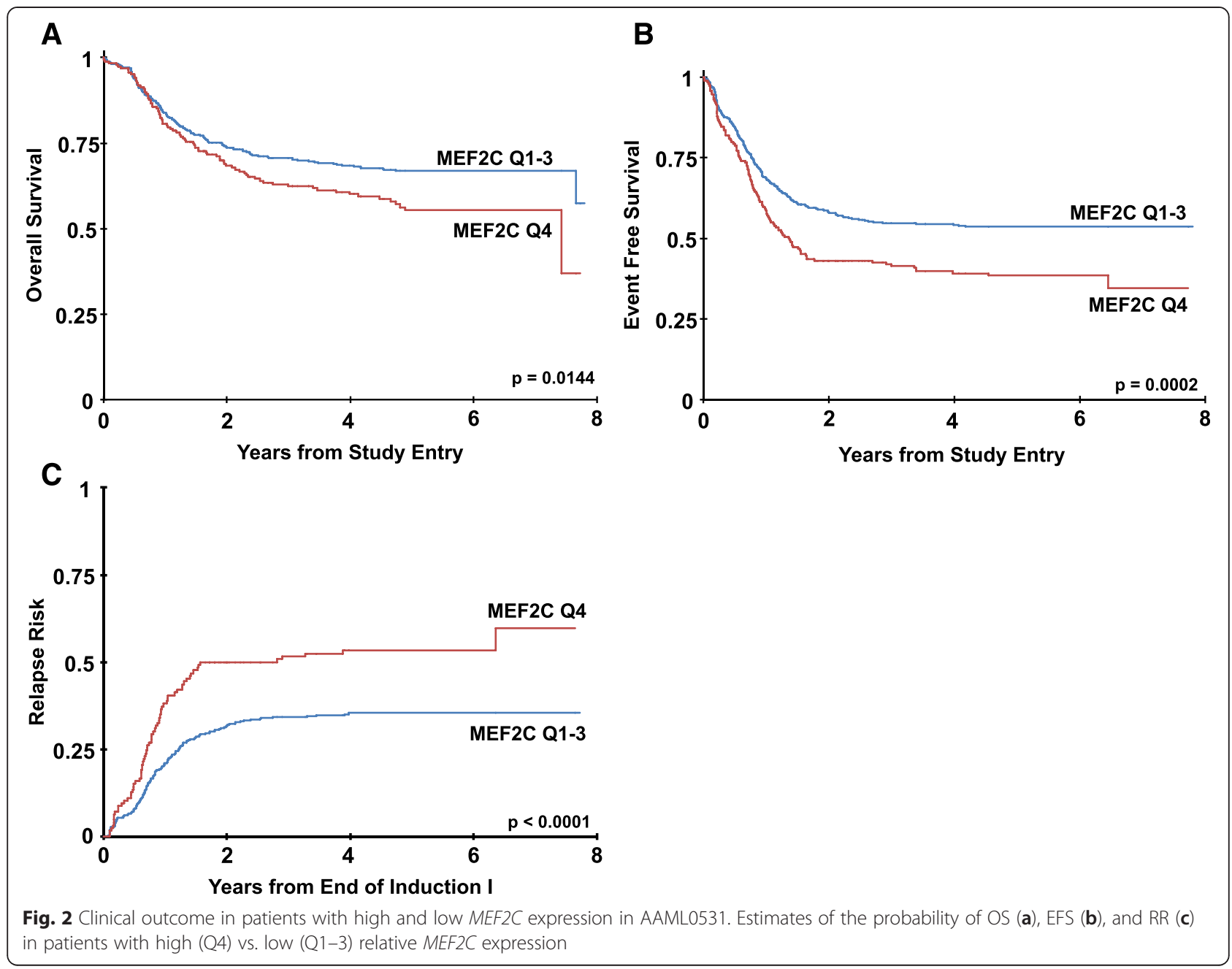

expression dominated and largely accounted for the association between $M E F 2 C$ expression and outcome. In fact, after multivariable adjustment, $M E F 2 C$ expression was not apparently associated with outcome. As $M E F 2 C$ expression does not provide prognostic information that is independent of established risk factors, $M E F 2 C$ may not be particularly useful as a response biomarker. Nonetheless, high $M E F 2 C$ expression was found to be associated with inferior efficacy of curative-intent, intensive AML chemotherapy. These data may, ultimately, provide a strong rationale for therapeutic targeting of $M E F 2 C$ transcriptional activation in this disease.

Because of the genetic, molecular, and immunophenotypic heterogeneity of human AML, identification of pharmacologic drugs suitable for reasonably large subsets of patients has remained challenging. Therefore, unraveling signaling aberrancies shared by many of the leukemias could be useful for the development of riskdirected, mechanism-based therapies. Our data suggest the possibility that targeting $M E F 2 C$-induced signaling could serve as one such strategy. Very recent studies have identified $M E F 2 C$ as a key factor in regulating suppressor of cytokine signaling-2 (SOCS2) in normal and malignant hematopoiesis and indicated that the MEF2C/ SOCS2 regulatory network might confer leukemic stemness features to a neoplastic hematopoietic clone [25]. Consistent with a close relationship between $M E F 2 C$ and SOCS2, we [26] and subsequently others [25] have provided evidence that high SOCS2 expression is associated with poor survival in AML. Studies in T-ALL and colon cancer cells have indicated that MEF2C may inhibit BCL2-regulated apoptosis and can function as a regulator of cell proliferation [7, 27]. A similar mechanism of apoptosis resistance induced by $M E F 2 C$ in AML cells may explain the apparent association between $M E F 2 C$ overexpression and failure of AML chemotherapy. Further experimental studies will be required to elucidate the mechanisms of $M E F 2 C$-induced leukemogenesis and effective therapeutic strategies to block them.

It is a strength of our analysis that we included a large number of diagnostic specimens from patients treated homogeneously on a recent cooperative group trial, 
Table 2 Comparison of baseline characteristics of patients with low (Q1-3) vs. high (Q4) MEF2C expression

\begin{tabular}{|c|c|c|c|}
\hline \multirow[t]{3}{*}{ Patient characteristics } & \multicolumn{2}{|c|}{ Relative MEF2C expression } & \multirow[t]{3}{*}{$P$ value } \\
\hline & Low (Q1-3) & High (Q4) & \\
\hline & $n=563$ & $n=188$ & \\
\hline Median age, years (range) & $10.55(0.01-29.8)$ & $6.9(0.06-19.8)$ & $<0.001$ \\
\hline Male sex, $n(\%)$ & $279(50 \%)$ & $95(51 \%)$ & 0.817 \\
\hline WBC $\left(\times 10^{3} / \mu \mathrm{L}\right)$, median (range) & $30.7(0.2-827.2)$ & $20.9(0.5-519.0)$ & 0.160 \\
\hline Median bone marrow blasts, $\%$ & $67.5(0-100)$ & $71(3-100)$ & 0.038 \\
\hline Platelet count $\left(\times 10^{3} / \mu \mathrm{L}\right)$, median (range) & $48(4-556)$ & $48(1-11,177)$ & 0.484 \\
\hline Hemoglobin (g/dL), median (range) & $8.1(2.3-17.0)$ & $8.1(1.8-17.0)$ & 0.684 \\
\hline \multicolumn{4}{|l|}{ Cytogenetics, $n(\%)$} \\
\hline Normal & $142(26 \%)$ & $21(12 \%)$ & $<0.001$ \\
\hline $\mathrm{t}(8 ; 21)(\mathrm{q} 22 ; \mathrm{q} 22)$ & $101(18 \%)$ & $10(6 \%)$ & $<0.001$ \\
\hline $\operatorname{inv}(16) / t(16 ; 16)(p 13.1 ; q 22)$ & $78(14 \%)$ & $12(7 \%)$ & 0.007 \\
\hline $\mathrm{t}(9 ; 11)(\mathrm{p} 22 ; q 23)$ or other abn 11q23 & $87(16 \%)$ & $67(37 \%)$ & $<0.001$ \\
\hline $\mathrm{t}(6 ; 9)(\mathrm{p} 23 ; \mathrm{q} 34)$ & $10(2 \%)$ & $1(1 \%)$ & 0.309 \\
\hline Monosomy 7 & $3(1 \%)$ & $11(6 \%)$ & $<0.001$ \\
\hline Del7q & $4(1 \%)$ & $2(1 \%)$ & 0.642 \\
\hline$-5 / 5 q-$ & $6(2 \%)$ & $4(2 \%)$ & 0.275 \\
\hline Trisomy 8 & $28(5 \%)$ & $19(10 \%)$ & 0.011 \\
\hline Other & $89(16 \%)$ & $34(19 \%)$ & 0.428 \\
\hline Unknown & 15 & 7 & \\
\hline \multicolumn{4}{|l|}{ Risk group, $n(\%)$} \\
\hline Standard & $230(42 \%)$ & $123(68 \%)$ & $<0.001$ \\
\hline Low & $255(46 \%)$ & $29(16 \%)$ & $<0.001$ \\
\hline High & $69(12 \%)$ & $30(16 \%)$ & 0.170 \\
\hline Unknown & 9 & 6 & \\
\hline \multicolumn{4}{|l|}{ Molecular alterations, \% } \\
\hline FLT3/ITD & $18 \%$ & $10 \%$ & 0.018 \\
\hline NPM1 mutation & $9 \%$ & $3 \%$ & 0.010 \\
\hline CEBPA mutation & $7 \%$ & $1 \%$ & 0.002 \\
\hline WT1 mutation & $6 \%$ & $5 \%$ & 0.688 \\
\hline Hepatomegaly, \% & $25 \%$ & $36 \%$ & 0.006 \\
\hline Splenomegaly, \% & $25 \%$ & $39 \%$ & $<0.001$ \\
\hline Extramedullary disease, \% & $15 \%$ & $11 \%$ & 0.196 \\
\hline CNS disease, $\%$ & $6 \%$ & $9 \%$ & 0.239 \\
\hline Chloroma, \% & $15 \%$ & $12 \%$ & 0.496 \\
\hline Treatment arm, $n(\%)$ & & & 0.689 \\
\hline Arm A—no GO & $281(50 \%)$ & $97(52 \%)$ & \\
\hline Arm B-with GO & $282(50 \%)$ & 91 (48 \%) & \\
\hline
\end{tabular}

thereby increasing the precision of the outcome estimates. On the other hand, our studies have some limitations that need to be acknowledged. First, despite the use of over 750 specimens, our study was not large enough to allow for extensive multivariate adjustments. Because of the sample size of the individual risk groups, our ability to perform subset analyses was similarly limited. Second, since unsorted bone marrow specimens were used for our studies, differences in MEF2C abundance between specimens may not necessarily reflect differences in AML blasts but, rather, other (i.e., nonleukemic) cells or varying compositions of less mature 
Table 3 Univariate and multivariate regression models of OS, EFS, and RR

\begin{tabular}{|c|c|c|c|c|c|c|c|c|c|c|c|c|}
\hline & \multicolumn{4}{|l|}{ OS } & \multicolumn{4}{|l|}{ EFS } & \multicolumn{4}{|l|}{$\mathrm{RR}$} \\
\hline & $n$ & $\mathrm{HR}$ & $95 \% \mathrm{Cl}$ & $P$ value & $n$ & $\mathrm{HR}$ & $95 \% \mathrm{Cl}$ & $P$ value & $n$ & $\mathrm{HR}$ & $95 \% \mathrm{Cl}$ & $P$ value \\
\hline \multicolumn{13}{|l|}{ Univariable model } \\
\hline \multicolumn{13}{|l|}{ MEF2C Expression } \\
\hline Low (Q1-3) & 563 & 1 & & & 563 & 1 & & & 430 & 1 & & \\
\hline High (Q4) & 188 & 1.385 & $1.07-1.80$ & 0.014 & 188 & 1.510 & $1.21-1.88$ & $<0.001$ & 126 & 1.813 & $1.36-2.42$ & $<0.001$ \\
\hline \multicolumn{13}{|l|}{ Disease risk ${ }^{a}$} \\
\hline Standard-risk & 353 & 1 & & & 353 & 1 & & & 254 & 1 & & \\
\hline Low-risk & 284 & 0.351 & $0.26-0.48$ & $<0.001$ & 284 & 0.440 & $0.34-0.56$ & $<0.001$ & 234 & 0.410 & $0.30-0.56$ & $<0.001$ \\
\hline High-risk & 99 & 1.234 & $0.90-1.69$ & 0.193 & 99 & 1.377 & $1.05-1.81$ & 0.022 & 57 & 0.886 & $0.59-1.34$ & 0.567 \\
\hline \multicolumn{13}{|l|}{ FAB Class } \\
\hline Not MO & 624 & 1 & & & 624 & 1 & & & 471 & 1 & & \\
\hline MO & 19 & 2.847 & $1.62-5.00$ & $<0.001$ & 19 & 2.192 & $1.33-3.63$ & 0.002 & 11 & 2.641 & $1.33-5.24$ & 0.006 \\
\hline \multicolumn{13}{|c|}{ Multivariable model $^{b}$} \\
\hline \multicolumn{13}{|c|}{ MEF2C Expression } \\
\hline Low (Q1-3) & 477 & 1 & & & 477 & 1 & & & 371 & 1 & & \\
\hline High (Q4) & 152 & 0.986 & $0.72-1.36$ & 0.929 & 152 & 1.135 & $0.86-1.49$ & 0.365 & 101 & 1.324 & $0.91-1.92$ & 0.137 \\
\hline \multicolumn{13}{|l|}{ Disease risk $^{a}$} \\
\hline Standard-risk & 292 & 1 & & & 292 & 1 & & & 213 & 1 & & \\
\hline Low-risk & 252 & 0.330 & $0.23-0.48$ & $<0.001$ & 252 & 0.419 & $0.31-0.56$ & $<0.001$ & 210 & 0.422 & $0.30-0.59$ & $<0.001$ \\
\hline High-risk & 85 & 1.251 & $0.85-1.78$ & 0.214 & 85 & 1.275 & $0.90-1.75$ & 0.180 & 49 & 0.872 & $0.56-1.36$ & 0.549 \\
\hline \multicolumn{13}{|l|}{ FAB Class } \\
\hline Not MO & 610 & 1 & & & 610 & 1 & & & 461 & 1 & & \\
\hline MO & 19 & 1.981 & $1.09-3.59$ & 0.024 & 19 & 2.382 & $1.05-5.42$ & 0.038 & 11 & 1.646 & $0.78-3.45$ & 0.187 \\
\hline
\end{tabular}

aSee "Methods" section for definition of cytogenetic/molecular disease risk

${ }^{\mathrm{b}}$ Models were also adjusted for treatment arm, FAB category (MO vs. no-M0), and age

and more mature AML cells. Gene expression studies in human material indicate that higher $M E F 2 C$ mRNA levels are found in less mature hematopoietic cells, including LSC populations [28, 29]. Additional studies will be required for the identification of the exact cellular origins of the greatly variable amounts of $M E F 2 C$ and more detailed analyses of relative expression levels along the cellular differentiation path of AML cells. Third, we only had cryopreserved specimens available for our analyses. Future studies will be necessary to determine to what degree, if any, $M E F 2 C$ expression changes in the cryopreservation process. And fourth, we were unable to formally study whether high $M E F 2 C$ mRNA expression leads to high MEF2C protein expression, a relationship that would provide a strong rationale for therapeutic targeting of MEF2C transcriptional activation in AML. However, preliminary data from ongoing laboratory studies indeed suggest that dysregulated $M E F 2 C$ transcription results in MEF2C protein overexpression and confers enhanced AML cell survival (A. Kentsis, personal communication). If clinically exploitable strategies to counteract MEF2C signaling were developed, it is conceivable that $M E F 2 C$ expression could become a biomarker of interest for successful drug development [30], e.g., to identify the subsets of patients most suitable for MEF2C-directed therapy.

\section{Conclusions}

Our data indicate that high $M E F 2 C$ expression identifies a subset of pediatric and adolescent AML patients with adverse-risk disease features and, consequently, significantly increased risk for primary treatment failure, relapse, and poor leukemia-free and overall survival. With confirmation that high $M E F 2 C$ mRNA expression leads to overexpression of MEF2C protein, these findings provide the rationale for therapeutic targeting of MEF2C transcriptional activation in AML.

\section{Methods}

\section{Patient samples}

Cryopreserved pretreatment ("diagnostic") specimens from patients enrolled in AAML0531 who consented to the biology studies and had bone marrow samples were 
available and were included in this study. The patient and disease (cytogenetic/molecular) characteristics of the subset of AAML0531 patients studied in this analysis were relatively comparable to patients not studied in this analysis. However, there were some differences in disease characteristics (i.e., higher proportion of patients with $\operatorname{inv}(16) / t(16 ; 16) \quad[P=0.007]$ and low-risk disease $[P<$ $0.001]$ ) as well as better short-term outcomes (i.e., $C R$ rate after one course of therapy $[P=0.005]$ albeit not rate of MRD $[P=0.132])$, but OS was similar $(P=0.52)$ and EFS was only slightly better $(P=0.04)$.

\section{Risk stratification}

A combination of cytogenetic and molecular abnormalities was used to stratify participants into risk groups. A patient was considered low-risk if a chromosomal abnormality/mutation was present in core binding factors (CBF; $\mathrm{t}(8 ; 21)$ or inv(16)/t $(16 ; 16)$ ), nucleophosmin (NPM1) (unless a FLT3/internal tandem duplication (ITD) mutation with high allelic ratio $[\geq 0.4]$ was also present), or $C E B P A$; for $C E B P A$, both single and double mutations were considered favorable [31]. Patients were classified as high-risk if they had monosomy 5 or deletion of $5 \mathrm{q}(-5 / 5 \mathrm{q}-)$, monosomy 7 (-7), or FLT3/ITD with high allelic ratio (0.4 or higher). All other patients with data sufficient for classification were considered standard-risk.

\section{Detection and quantification of minimal residual disease (MRD)}

Residual AML was quantified in bone marrow aspirates collected at the end of the first induction course by multiparameter flow cytometry using a "different-from-normal" approach as previously described [32].

\section{Quantification of MEF2C expression in unsorted AML specimens}

Total RNA from unsorted diagnostic AML specimens was extracted with the AllPrep DNA/RNA Mini Kit using the QIAcube automated system (Qiagen, Valencia, CA). After quantification with a microvolume spectrophotometer (NanoDrop; Thermo Scientific, Wilmington, $\mathrm{DE}), 10 \mathrm{ng}$ of total RNA was subjected to quantitative reverse-transcriptase polymerase chain reaction (qRTPCR; 7900 Real-Time PCR System; Applied Biosystems; Foster City, CA) using TaqMan primers per manufacturer's instructions to determine expression of $M E F 2 C$ and, for normalization, the housekeeping gene, GUSB. Primer probe sets were as follows: $M E F 2 C$ was designed to amplify sequence at the junction of exons 6 and 7 , and GUSB was designed to amplify sequence at the junction of exons 8 and 9 (Hs00231149_m1 and Hs00939627_ $\mathrm{m} 1$, respectively; Applied Biosystems). Patient samples were run in duplicate, and the $\Delta \Delta C T$ method quantified as $2^{(-\Delta \Delta C T)}[33,34]$ was used to determine the expression levels of $M E F 2 C$ relative to GUSB.

\section{Statistical analysis}

Data from AAML0531 were current as of December 31, 2013. The median (range) of follow-up for patients alive at last contact was $4.3(0.02-7.1)$ years. The KaplanMeier method [35] was used to estimate OS (defined as time from study entry to death) and EFS (time from study entry until failure to achieve CR during induction, relapse, or death). RR was calculated by cumulative incidence methods defined as time from the end of induction I for patients in CR to relapse or death where deaths without a relapse were considered competing events [36]. Patients who withdrew from therapy due to relapse, persistent central nervous system (CNS) disease, or refractory disease with $>20 \%$ bone marrow blasts by the end of induction I were defined as induction I failures. The significance of predictor variables was tested with the log-rank statistic for OS and EFS and with Gray's statistic for RR. All estimates are reported with two times the Greenwood standard errors. Children lost to follow-up were censored at their date of last known contact. Cox proportional hazards models [37] were used to estimate the hazard ratio (HR) for defined groups of patients in univariate and multivariate analyses of OS and EFS. Analyses of univariable OS for low-risk patients and multivariable EFS for all patients violated the proportional hazards assumption, and therefore, a parametric cure regression model was used to estimate the HR. Competing risk regression models were used to estimate HRs for univariate and multivariate analyses of $R R$. The chi-square test was used to test the significance of observed differences in proportions, and Fisher's exact test was used when data were sparse. Differences in medians were compared by the Mann-Whitney or Wilcoxon signed-rank tests as appropriate. A $P$ value $<0.05$ was considered statistically significant.

\section{Ethics, consent, and permissions}

Informed consent was obtained from all study subjects in accordance with the Declaration of Helsinki, and the institutional review boards (IRBs) of all participating institutions approved the clinical protocol. IRB approval was obtained from Fred Hutchinson Cancer Research Center before conduct of this biological study, which was also approved by the COG Myeloid Disease Biology Committee and the National Cancer Institute Cancer Therapy Evaluation Program.

Competing interests

The authors have declared no conflicts of interests. 


\section{Authors' contributions}

GSL and RBW designed and performed research, analyzed and interpreted data, and wrote the manuscript. TAA, RBG, and Y-CW performed statistical analyses and analyzed interpreted data. CJG, KHH, and RER performed research and wrote the manuscript. AK analyzed and interpreted data and wrote the manuscript. SCR, BAH, ASG, and SM collected, analyzed, and interpreted data. All authors revised the manuscript critically, and gave final approval to submit for publication.

\section{Acknowledgements}

We thank the COG AML Reference Laboratory for providing diagnostic AML specimens.

\section{Financial support}

This work was supported by grants R21-CA161894, U10-CA098543, U10-CA180899, and U24-CA114766 from the National Cancer Institute/ National Institutes of Health, Bethesda, MD, USA. R.B.W. is a Leukemia \& Lymphoma Scholar in Clinical Research. The funding sources played no role in the design; in the collection, analysis, and interpretation of data; in the writing of the manuscript; and in the decision to submit the manuscript for publication.

\section{Author details}

${ }^{1}$ Clinical Research Division, Fred Hutchinson Cancer Research Center, 1100 Fairview Ave N, D2-190, Seattle, WA 98109-1024, USA. ²Department of Biostatistics, University of Southern California, Los Angeles, CA, USA. ${ }^{3}$ Children's Oncology Group, Monrovia, CA, USA. ${ }^{4}$ Molecular Pharmacology and Chemistry Program, Sloan Kettering Institute, New York, NY, USA. ${ }^{5}$ Department of Pediatrics, Memorial Sloan Kettering Cancer Center, New York, NY, USA. 'Weill Medical College of Cornell University, New York, NY, USA. ${ }^{7}$ Department of Pathology, St. Jude Children's Research Hospital, Memphis, TN, USA. ${ }^{8}$ Department of Laboratory Medicine and Pathology, University of Minnesota Cancer Center, Minneapolis, MN, USA. 'Division of Hematology-Oncology, Children's Mercy Hospitals and Clinics, Kansas City, MO, USA. ${ }^{10}$ Department of Pediatrics, University of Washington, Seattle, WA USA. ${ }^{11}$ Department of Medicine, Division of Hematology, University of Washington, Seattle, WA, USA. ${ }^{12}$ Department of Epidemiology, University of Washington, Seattle, WA, USA.

\section{Received: 24 August 2015 Accepted: 13 October 2015} Published online: 20 October 2015

\section{References}

1. Black BL, Olson EN. Transcriptional control of muscle development by myocyte enhancer factor-2 (MEF2) proteins. Annu Rev Cell Dev Biol. 1998;14:167-96.

2. Lin Q, Schwarz J, Bucana C, Olson EN. Control of mouse cardiac morphogenesis and myogenesis by transcription factor MEF2C. Science. 1997;276(5317):1404-7.

3. Canté-Barrett K, Pieters R, Meijerink JP. Myocyte enhancer factor $2 \mathrm{C}$ in hematopoiesis and leukemia. Oncogene. 2014;33(4):403-10.

4. Schüler A, Schwieger M, Engelmann A, Weber K, Horn S, Müller U, et al. The MADS transcription factor Mef2c is a pivotal modulator of myeloid cell fate. Blood. 2008;111(9):4532-41.

5. Stehling-Sun S, Dade J, Nutt SL, DeKoter RP, Camargo FD. Regulation of lymphoid versus myeloid fate 'choice' by the transcription factor Mef2c. Nat Immunol. 2009;10(3):289-96.

6. Zheng R, Wang X, Studzinski GP. 1,25-Dihydroxyvitamin D3 induces monocytic differentiation of human myeloid leukemia cells by regulating C/EBPbeta expression through MEF2C. J Steroid Biochem Mol Biol. 2015;148:132-7.

7. Nagel S, Meyer C, Quentmeier H, Kaufmann M, Drexler HG, MacLeod RA. MEF2C is activated by multiple mechanisms in a subset of T-acute lymphoblastic leukemia cell lines. Leukemia. 2008:22(3):600-7.

8. Nagel S, Venturini L, Meyer C, Kaufmann M, Scherr M, Drexler HG, et al. Transcriptional deregulation of oncogenic myocyte enhancer factor $2 \mathrm{C}$ in T-cell acute lymphoblastic leukemia. Leuk Lymphoma. 2011;52(2):290-7.

9. Homminga I, Pieters R, Langerak AW, de Rooi JJ, Stubbs A, Verstegen M, et al. Integrated transcript and genome analyses reveal NKX2-1 and MEF2C as potential oncogenes in T cell acute lymphoblastic leukemia. Cancer Cell. 2011;19(4):484-97.
10. Zuurbier L, Gutierrez A, Mullighan CG, Cante-Barrett K, Gevaert AO, de Rooi J, et al. Immature MEF2C-dysregulated T-cell leukemia patients have an early T-cell precursor acute lymphoblastic leukemia gene signature and typically have non-rearranged T-cell receptors. Haematologica. 2014;99(1):94-102.

11. Schwieger M, Schüler A, Forster M, Engelmann A, Arnold MA, Delwel R, et al. Homing and invasiveness of MLL/ENL leukemic cells is regulated by MEF2C. Blood. 2009;114(12):2476-88.

12. Somervaille TCP, Cleary ML. Grist for the MLL: how do MLL oncogenic fusion proteins generate leukemia stem cells? Int J Hematol. 2010;91(5):735-41.

13. de Boer J, Walf-Vorderwulbecke V, Williams O. In focus: MLL-rearranged leukemia. Leukemia. 2013;27(6):1224-8.

14. Gole B, Wiesmüller L. Leukemogenic rearrangements at the mixed lineage leukemia gene (MLL)-multiple rather than a single mechanism. Front Cell Dev Biol. 2015:3:41.

15. Yokoyama A. Molecular mechanisms of MLL-associated leukemia. Int J Hematol. 2015;101(4):352-61.

16. Jin G, Yamazaki Y, Takuwa M, Takahara T, Kaneko K, Kuwata T, et al. Trib1 and Evi1 cooperate with Hoxa and Meis1 in myeloid leukemogenesis. Blood. 2007;109(9):3998-4005

17. Yoshimi A, Goyama S, Watanabe-Okochi N, Yoshiki Y, Nannya Y, Nitta E, et al. Evi1 represses PTEN expression and activates PI3K/AKT/mTOR via interactions with polycomb proteins. Blood. 2011;117(13):3617-28.

18. Glass C, Wilson M, Gonzalez R, Zhang Y, Perkins AS. The role of EVI1 in myeloid malignancies. Blood Cells Mol Dis. 2014;53(1-2):67-76.

19. Bindels EM, Havermans M, Lugthart S, Erpelinck C, Wocjtowicz E, Krivtsov $A V$, et al. EVI1 is critical for the pathogenesis of a subset of MLL-AF9rearranged AMLs. Blood. 2012;119(24):5838-49.

20. Rommer A, Steinmetz B, Herbst F, Hackl H, Heffeter P, Heilos D, et al. EVI1 inhibits apoptosis induced by antileukemic drugs via upregulation of CDKN1A/p21/WAF in human myeloid cells. PLoS One. 2013;8(2):e56308.

21. Heller G, Rommer A, Steinleitner K, Etzler J, Hackl H, Heffeter P, et al. EVI1 promotes tumor growth via transcriptional repression of MS4A3. J Hematol Oncol. 2015;8:28

22. Du Y, Spence SE, Jenkins NA, Copeland NG. Cooperating cancer-gene identification through oncogenic-retrovirus-induced insertional mutagenesis. Blood. 2005;106(7):2498-505.

23. Krivtsov AV, Twomey D, Feng Z, Stubbs MC, Wang Y, Faber J, et al. Transformation from committed progenitor to leukaemia stem cell initiated by MLL-AF9. Nature. 2006;442(7104):818-22.

24. Gamis AS, Alonzo TA, Meshinchi S, Sung L, Gerbing RB, Raimondi SC, et al. Gemtuzumab ozogamicin in children and adolescents with de novo acute myeloid leukemia improves event-free survival by reducing relapse risk: results from the randomized phase III Children's Oncology Group trial AAML0531. J Clin Oncol. 2014;32(27):3021-32.

25. Vitali C, Bassani C, Chiodoni C, Fellini E, Guarnotta C, Miotti S, et al. SOCS2 controls proliferation and stemness of hematopoietic cells under stress conditions and its deregulation marks unfavorable acute leukemias. Cancer Res. 2015;75(11):2387-99.

26. Laszlo GS, Ries RE, Gudgeon CJ, Harrington KH, Alonzo TA, Gerbing RB, et al. High expression of suppressor of cytokine signaling-2 predicts poor outcome in pediatric acute myeloid leukemia: a report from the Children's Oncology Group. Leuk Lymphoma. 2014;55(12):2817-21.

27. Badodi S, Baruffaldi F, Ganassi M, Battini R, Molinari S. Phosphorylationdependent degradation of MEF2C contributes to regulate G2/M transition. Cell Cycle. 2015;14(10):1517-28

28. Seita J, Sahoo D, Rossi DJ, Bhattacharya D, Serwold T, Inlay MA, et al. Gene Expression Commons: an open platform for absolute gene expression profiling. PLoS One. 2012;7(7):e40321.

29. Gentles AJ, Plevritis SK, Majeti R, Alizadeh AA. Association of a leukemic stem cell gene expression signature with clinical outcomes in acute myeloid leukemia. JAMA. 2010;304(24):2706-15.

30. Smith AD, Roda D, Yap TA. Strategies for modern biomarker and drug development in oncology. J Hematol Oncol. 2014;7(1):70.

31. Ho PA, Alonzo TA, Gerbing RB, Pollard J, Stirewalt DL, Hurwitz C, et al. Prevalence and prognostic implications of CEBPA mutations in pediatric acute myeloid leukemia (AML): a report from the Children's Oncology Group. Blood. 2009;113(26):6558-66.

32. Loken MR, Alonzo TA, Pardo L, Gerbing RB, Raimondi SC, Hirsch BA, et al. Residual disease detected by multidimensional flow cytometry signifies 
high relapse risk in patients with de novo acute myeloid leukemia: a report from Children's Oncology Group. Blood. 2012;120(8):1581-8.

33. Livak KJ, Schmittgen TD. Analysis of relative gene expression data using real-time quantitative PCR and the 2(-Delta Delta C(T)) Method. Methods. 2001;25(4):402-8.

34. Schmittgen TD, Livak KJ. Analyzing real-time PCR data by the comparative C(T) method. Nat Protoc. 2008;3(6):1101-8.

35. Kaplan EL, Meier P. Nonparametric estimation from incomplete observations. J Am Stat Assoc. 1958;53(282):457-81.

36. Kalbfleisch JD, Prentice RL. The statistical analysis of failure time data. Hoboken, NJ: John Wiley \& Sons, Inc.; 2002.

37. Cox DR. Regression models and life tables (with discussion). J R Statist Soc 1972;34(B):187-220.

\section{Submit your next manuscript to BioMed Central} and take full advantage of:

- Convenient online submission

- Thorough peer review

- No space constraints or color figure charges

- Immediate publication on acceptance

- Inclusion in PubMed, CAS, Scopus and Google Scholar

- Research which is freely available for redistribution 